\title{
Sex-biased admixture and assortative mating shape genetic variation and influence demographic inference in admixed Cabo Verdeans
}

Katharine L Korunes, ${ }^{1}$ Giordano Bruno Soares-Souza, ${ }^{2}$ Katherine Bobrek, ${ }^{3}$ Hua Tang, ${ }^{4}$ Isabel Inês Araújo, ${ }^{5}$ Amy Goldberg, ${ }^{* 1,6}$ Sandra Beleza**2,6

1) Evolutionary Anthropology, Duke University, Durham, North Carolina, 27705, USA

2) Department of Genetics and Genome Biology, University of Leicester, Leicester, LE1 7RH, United Kingdom

3) Department of Anthropology, Emory University, Atlanta, Georgia, 30322, USA

4) Department of Genetics, Stanford University School of Medicine, Stanford, California, 94305 USA

5) Faculdade de Ciências e Tecnologia, Universidade de Cabo Verde (Uni-CV), Praia, Ilha de Santiago, Cabo Verde

6) These authors contributed equally to this work

*Correspondence: amy.goldberg@duke.edu

${ }^{* *}$ Correspondence: sdsb1@leicester.ac.uk 


\section{Abstract}

2 Genetic data can provide insights into population history, but first we must understand the

3 patterns that complex histories leave in genomes. Here, we consider the admixed human

4 population of Cabo Verde to understand the patterns of genetic variation left by social and

5 demographic processes. First settled in the late 1400s, Cabo Verdeans are admixed descendants

6 of Portuguese colonizers and enslaved West African people. We consider Cabo Verde's well-

$7 \quad$ studied historical record alongside genome-wide SNP data from 563 individuals from 4 regions

8 within the archipelago. We use genetic ancestry to test for patterns of nonrandom mating and

9 sex-specific gene flow, and we examine the consequences of these processes for common

10 demographic inference methods and for genetic patterns. Notably, multiple population genetic

11 tools that assume random mating underestimate the timing of admixture, but incorporating non-random mating produces estimates more consistent with historical records. We consider

13 how admixture interrupts common summaries of genomic variation such as runs-of-

14 homozygosity $(\mathrm{ROH})$. While summaries of $\mathrm{ROH}$ may be difficult to interpret in admixed

15 populations, differentiating $\mathrm{ROH}$ by length class shows that $\mathrm{ROH}$ reflect historical differences

16 between the islands in their contributions from the source populations and post-admixture

17 population dynamics. Finally, we find higher African ancestry on the $\mathrm{X}$ chromosome than on

18 the autosomes, consistent with an excess of European males and African females contributing to

19 the gene pool. Considering these genomic insights into population history in the context of

20 Cabo Verde's historical record, we can identify how assumptions in genetic models impact

21 inference of population history more broadly. 


\section{Author Summary}

23 Patterns of genetic variation are often used to infer human population histories; however

24 population-genetic models make a variety of simplifying assumptions that often neglect the

25 demographic and social dynamics of human populations. Here, we use the population of Cabo

26 Verde as a case study to understand the patterns of genetic variation left by social and

27 demographic processes. The islands of Cabo Verde were first settled in the late 1400s by

28 Portuguese colonizers and enslaved West African people. We consider genomic data from four

29 regions within the archipelago alongside historical records that document settlement patterns,

30 timing, and sociocultural dynamics within the islands. We use genetic ancestry to test for

31 nonrandom mating and sex-specific demography. We show that these sociocultural processes

32 may bias inference of population history parameters and distributions of shared ancestry in the

33 region. Overall, by providing insights into the patterns of genetic variation social processes

34 leave in human genomes for a population with a well-studied historical record, we highlight

35 processes to consider in order to more accurately understand the history of populations without

36 extensive records.

\section{Introduction}

38 Patterns of genetic variation are often used to infer human population histories, such as

39 fluctuations in population size, the timing of gene flow, and changes in population structure (1-

40 7). These studies have been informative about ancient and modern populations that may lack 
41 historical records (8-12). However, by necessity, population-genetic models make a variety of

42 simplifying assumptions that often neglect the demographic and social dynamics of human

43 populations. For example, many classical models assume random mating, but within empirical

44 populations mating is often nonrandom, known as assortative mating. Positive assortative

mating in human populations can keep haplotype blocks together, affecting evolutionary

processes and our ability to infer population history (13-15). Further, male and female

demographic processes can differ and vary over time. Comparisons between the $X$

chromosome and autosomes have shown that population sizes and migration rates differ between males and females of many human populations (16-20).

The challenges of inferring human demographic history are particularly apparent on short timescales of tens of generations, where changes in allele frequencies may be difficult to observe and biased by dynamics not captured by classic models. Admixed populations provide an opportunity to examine evolutionary processes on short timescales using admixture linkagedisequilibrium (LD) structure rather than potentially small changes in allele frequencies. Understanding the patterns generating the distribution of ancestry within an admixed population is also important as genomic data has shown how widespread admixture is throughout human evolution $(8,11,21-26)$.

In admixed populations, nonrandom mating can be driven by factors that correlate with genetic ancestry. For example, spouse pairs within multiple Latino populations are correlated in their genomic ancestry $(13,15)$. When mating patterns lead to a positive correlation in genomic 
ancestry, this specific type of nonrandom mating, referred to as ancestry-assortative mating, can increase the likelihood that individuals in mating pairs share recent common ancestors. Recent work has begun to assess the implications of these patterns, including effects on common demographic inference strategies and on genetic/phenotypic variation $(7,14,24)$. One genetic consequence of positive ancestry-assortative mating is an increase in long runs of homozygosity $(\mathrm{ROH})$, which arise when long tracts of homozygous genotypes occur due to inheritance of identical by descent (IBD) haplotypes from recent common ancestors. Across modern human populations, $\mathrm{ROH}$ are common and typically range in length from kilobases to megabases (2730). In the field of population genetics, we are just beginning to learn about ROH dynamics and expectations in admixed populations, which do not necessarily fit expectations built from homogenous populations (30,31). For example, we know that distinct length classes of $\mathrm{ROH}$ reflect demographic factors from different timescales, but as we explore here, it is unclear what these distributions of $\mathrm{ROH}$ mean in populations that have undergone major demographic transitions such as recent admixture. Patterns of genetic variation can also be shaped by differences in male and female demographic processes. In many human populations, population sizes and migration rates differ between males and females, leading to differences in ancestry patterns on the $\mathrm{X}$ chromosome vs the autosomes (16-20).

Here, we use the admixed population of Cabo Verde as a case study to understand the patterns of genetic variation left by multifaceted social and demographic processes in humans. Admixed populations of African ancestry, such as the population of Cabo Verde, are often excluded in the context of medical genetics and human evolutionary genetics, despite their importance and 
widespread global presence (32-34). The record of Cabo Verde population history is vast, including royal charters, letters from Crown officials, captains and other sea explorers' journals, records of the economic activities in Cabo Verde (e.g., landowners' rents and number of enslaved individuals traded), detailed ship logs of trade activity, and church censuses from the $18^{\text {th }}$ century on, which allows us to generate realistic assumptions about colonization and demography (35-41). We consider patterns of genetic variation alongside historical records that document many aspects of Cabo Verdean history, including settlement patterns, timing, and sociocultural dynamics. Specifically, we use distributions of genetic ancestry to test for ancestryassortative mating and sex-specific gene flow. We examine the consequences of these processes for genetic variation, such as patterns of homozygosity, and for common demographic inference methods. In turn, by elucidating the patterns of genetic variation social processes leave in human genomes for a population with a well-studied historical and ethnographic record, we can better use population-genomic methods to explore the history of populations without extensive records.

\section{Cabo Verdean history}

The recently admixed human population of Cabo Verde presents a particularly relevant opportunity to examine how sex-biased admixture and nonrandom mating shape genetic patterns and influence demographic inference. Cabo Verde is an archipelago off the coast of Senegal, inhabited today by admixed descendants of Portuguese colonizers and enslaved West African people who settled the unpopulated islands beginning in the mid-1400s (42-45). Since the archipelago was unoccupied prior to the 1460s, we have historical knowledge of the start of 
106 admixture and the contributing source populations, with written records clearly documenting

107 arrival times by island. Additionally, the island geography imposes population structure within

108 Cabo Verde, and historical records document differences in population sizes, mating patterns,

109 and social customs by island population (see Methods for historical data sources).

111 The settlement of the islands was influenced by island geography and ecology, and is often

112 divided into three different temporal stages, which are associated with changes in the economy

113 (35). The initial settlement stage (beginning in the 1460s) corresponds to the peopling of the

114 Southern island Santiago, followed by the nearby island of Fogo 20 years later (Fig 1). During

115 this time, the economy was centered around large cotton plantations and expanding trade with

116 the Senegambian coast. This trade included the exportation of great numbers of enslaved

117 Africans to Cabo Verde, the majority of which became part of the commercial trade to the

118 Americas $(36,37)$. In the $17^{\text {th }}$ century, following the decline of the slavery-based economy of

119 Santiago and Fogo, many free farmers seeking better farming conditions migrated to the

northwestern islands of Santo Antão and São Nicolau. These migrations comprised the second

121 settlement stage. In contrast to Santiago and Fogo, these northwestern islands did not become

122 populous economic centers (35). During the third and final settlement stage, São Vicente in the

123 northwestern group of islands was colonized by settlers from the neighboring islands in the

124 mid $19^{\text {th }}$ century. With the opening of Mindelo's harbor at that time, São Vicente prospered

125 from the advent of commercial Atlantic shipping, becoming the second most important island

126 of the archipelago after Santiago in terms of population size. In the $19^{\text {th }}$ century, the eastern 
127

128

129

130

131

132

133

134

135

136

137

138

139

140

141

142

143

144

145

146

islands Sal, Boa Vista, and Maio were also open to English and North American ships, but these islands never became densely populated $(35,37)$.

Previous investigations of genetic ancestry in Cabo Verde $(42,43,45)$ have shown extensive admixture in the archipelago and differences in the mean African genetic ancestry across islands, which may reflect the differences in the settlement history and suggest restricted gene

flow between islands. These past studies of Cabo Verdean genetic admixture have explored how genetic ancestry connects to phenotypic variation such as skin and eye color $(42,43)$, and to cultural information such as linguistic variation (45). Some earlier analyses of post-admixture population structure were based on Y-chromosome diversity (35), giving insight into malespecific demographic processes. Here, we examine how historical and social processes influence genetic variation and population-genetic inference of demography. We consider how the distributions of segments of DNA shared identically by descent within and among individuals (as measured by IBD, kinship, and $\mathrm{ROH}$ ) relate to patterns of genetic ancestry in an admixed population. We integrate several population genomic approaches using autosomal and Xchromosome ancestry and genetic variation patterns to infer the sex-specific demographic history of the last $\sim 20$ generations since founding, and we consider the potential biases of ancestry-assortative mating on the inference of admixture timing. Overall, we provide insights into the population history of Cabo Verde and demonstrate how admixed populations can provide powerful test cases for understanding evolutionary processes on short timescales. 


\section{Patterns of shared ancestry reflect the colonization history of the islands}

São Vicente Island. 
168 The process of admixture can influence measures such as IBD and $\mathrm{ROH}$ that are often used to

169 inform inference of population history, and theoretical expectations for these measures are less

170 clear for admixed populations compared to homogeneous populations. Thus, we use multiple

171 methods to examine relatedness in Cabo Verde, and we use this case study to underscore the

172 need for further empirical and theoretical work to understand the dynamics of IBD and ROH in

173 admixed populations. Patterns of IBD within and between populations provide opportunities to

174 examine common ancestry based on the number and sizes of segments of IBD. Using these

175 summaries of IBD to examine relatedness between and within regions of Cabo Verde, we found

176 that patterns of shared ancestry reflect the successive settlement history of the islands. Notably,

177 Santiago has the lowest mean number and total length of IBD segments between individuals

178 (Supp Fig 4-5). In contrast, we observe the highest levels of IBD within and between the

179 Northwest Cluster and Boa Vista.

180

181 To summarize patterns of IBD within and between the four island regions, we built a network

182 of relationships with pairs of individuals connected if they have IBD levels in the top 3\% of the

183 Cabo Verde IBD distribution (Fig 1; see Supp Fig 3 for lower levels of IBD and within-island

184 networks). In a non-admixed population, these edges roughly correspond to $4^{\text {th }}$-degree relatives

185 or closer (e.g., great-great-grandparents, great-great-grandchildren, great-aunts/uncles, or

186 grand-nieces/nephews). At this level of relatedness, we find that the presence of pairs of

187 individuals within and between islands connected by an edge is common, suggestive of recent

188 relatives both within and across islands. However, relatedness parameters estimated under 
189

190

191

192

193

194

195

196

197

198

199

200

201

202

203

simple models of structure likely do not hold in many human populations, such as recently

admixed populations. Kinship estimated under a framework (46) specifically designed for

arbitrary population structures (Supp Fig 6A) is consistent with both the overall trends in IBD

tracts (Supp Fig 4-5), and supports the presence of a subset of very closely related individuals within the data.

The lower levels of IBD (Fig 1, Supp Fig 3-5) and lower kinship estimates (Supp Fig 6) observed in Santiago are consistent with expectations based on the island's history, as Santiago was the

first Cabo Verdean island to be founded and has the largest population size. Santiago is also likely to have relatively high genetic diversity as a result of having the highest proportion of

West African ancestry, based on the expectation that kinship in human populations generally reflects serial bottlenecks due to dispersal from Africa $(47,48)$. In contrast, the high levels of IBD within and between the Northwest Cluster and Boa Vista reflect well-documented serial founding and migrations during the settlement of the islands. In general, kinship estimates also reflect the colonization history of the islands, with Cabo Verdeans of greater European ancestry sharing greater kinship compared to individuals with greater West African ancestry (Supp Fig 6). Exceptions to this trend were most noticeable in individuals from Fogo and the Northwest Cluster, where it was most apparent that some observed relatedness patterns do not result in smooth ordering of global ancestry proportions (Supp Fig 6B). This observation led us to hypothesize that other sociocultural processes beyond proportions of ancestry, such as nonrandom mating, may drive patterns of relatedness in Cabo Verde. 


\section{Nonrandom mating shapes patterns of ancestry and influences}

\section{1 demographic inference}

212 To test for ancestry assortative mating within Cabo Verde, we examined whether the genomic

213 ancestries of individuals in mating pairs correlate with each other. To this end, we applied

ANCESTOR to computationally infer the parental ancestry proportions that likely preceded the

independently to prevent uncertainty introduced by matching interchromosomal haplotypes.

217 As an example, the inferred ancestries of the parental haplotypes that likely preceded

chromosome 7 are significantly positively correlated for all islands except for Boa Vista (Fig 2A).

We observed similar results across the full set of autosomes (Fig 2B). We found that the inferred

ancestries of mating pairs in the previous generation are positively correlated, and that these

correlations differ significantly from expectations under random mating (random sampling

empirical distributions of parental haplotypes into pairs, shown in Supp Fig 7).

We next considered how the observed genetic evidence of assortative mating can be leveraged

in the context of inferring population history. To estimate the timing of the onset of admixture,

we applied the method of Zaitlen et al. (2017) (7), which describes the decay of local ancestry 
231 older estimates of admixture timing under a model including both assortative mating and

232 constant migration (Fig 3, Supp Fig 8), with increasingly older timing estimates as assortative strong ancestry-assortative mating, the models that considered substantial ancestry-assortative historical estimates (Fig 3, Supp Fig 8).

We additionally applied two strategies, ALDER (4) and MultiWaver 2.0 (5), that are not intended to account for assortative mating. ALDER uses the extent of LD decay among neighboring loci to infer mixture proportions and dates. In contrast, MultiWaver uses the length with ALDER suggest admixture timing in the mid to late 1700s (Fig 3; all timing estimates are

The estimates of admixture timing that do not explicitly consider ancestry-assortative mating, 
assortative mating and constant migration (Fig 3, Supp Fig 8). While multiple complex

demographic factors likely impact these estimates of admixture timing (see "Patterns of genetic

variation and admixture in Cabo Verde influence demographic inference" in the Discussion),

the evidence of ancestry-assortative mating in Cabo Verde (Fig 2, Supp Fig 7) and the LAD-

based inferences incorporating assortative mating (Fig 3, Supp Fig 8) suggest that explicitly

accounting for ancestry-assortative mating improves estimates of admixture timing, while the

assumption of random mating leads to underestimation of the age of admixture.

\section{Runs-of-homozygosity ( $\mathrm{ROH})$ reflect the contributions of the source}

\section{populations and patterns of nonrandom mating.}

262 We identified $\mathrm{ROH}$ using the method of Pemberton et al. (2012) as implemented in the software

263 GARLIC (30,49). This method classifies ROH into three categories (short, medium, and long)

264 based on the modeling of the length distribution in each population as a mixture of Gaussian

265 distributions. $\mathrm{ROH}$ arise when IBD haplotypes are inherited from a common ancestor. Thus,

266 distinct length classes reflect inheritance from ancestors at different timescales in the

267 population's history. We examined the distributions of total $\mathrm{ROH}$ per genome (sum of $\mathrm{ROH}$

268 segments of a specific length) within Cabo Verde, partitioning the distributions into length

269 classes that are enriched for pre- and post-colonization processes. Under the ROH classification

270 model of GARLIC, short and medium $\mathrm{ROH}$ formed due to processes that largely occurred

271 within the source populations prior to the founding of Cabo Verde. Thus, while $\mathrm{ROH}$ in non-

272 admixed populations are often modeled as three length classes, we emphasize the differences in 
pre- and post-admixture dynamics in Cabo Verde by focusing on shorter $\mathrm{ROH}$ (comprising both short and medium as called with GARLIC) vs. long ROH (Fig 4A-B). To ensure our results were consistent across the four classification schemes (Supp Fig 9).

Since methods for detecting and interpreting $\mathrm{ROH}$ have been developed primarily in nonadmixed populations $(30,50)$, we consider $\mathrm{ROH}$ in Cabo Verde as an example of $\mathrm{ROH}$ distributions in admixed populations where we can explore results in the context of ancestry and population history. For example, to test for genetic evidence that population bottlenecks contributed to observed patterns, we estimated ancestry-specific population sizes (Supp Fig 10). Ancestry-specific population sizes in Cabo Verde suggest bottlenecks of both source populations, followed by population expansion within the past 10 generations $(1,2)$. If the 
295 Comparing the Cabo Verdean islands to each other, we observed the lowest levels of total $\mathrm{ROH}$

296 in all length classes of ROH in Santiago (pairwise Mann-Whitney $U$ tests $\mathrm{p}<1 \times 10^{-8}$ ). The low

297 amounts of all length classes of $\mathrm{ROH}$ in Santiago are consistent with several historical attributes

298 of the islands, including serial founding beginning with the settlement of Santiago. Serial

299 founding resulting in bottlenecks is expected to increase shorter $\mathrm{ROH}$ by reducing the number

300 of ancestral lineages. But because shorter $\mathrm{ROH}$ reflects background relatedness from the source

301 populations, shorter $\mathrm{ROH}$ are expected to be more sensitive to the contributions of the source

302 populations.

304 Despite recent colonization bottlenecks, we observed that some admixed individuals in Cabo

305 Verde present lower levels of $\mathrm{ROH}$ per genome than either of the source population proxies.

306 Specifically, our classification of $\mathrm{ROH}$ into shorter and long $\mathrm{ROH}$ (Fig 4; Supp Fig 9) revealed

that Santiago has significantly lower levels of shorter ROH compared the West African

308 reference population (Mann-Whitney $\mathrm{U} p=5.87 \times 10^{-7}$ ), while long $\mathrm{ROH}$ are not depleted

309 (Mann-Whitney U p = 0.841). This observation that Santiago has significantly lower levels of

310 shorter $\mathrm{ROH}$ compared to even the West African reference is perhaps surprising given the

311 canonical view of African source populations as generically having the lowest $\mathrm{ROH}$ in

312 worldwide samples. This is consistent with the idea that shorter $\mathrm{ROH}$ are contributed to the

313 population from older shared ancestors in the source populations, and that these tracts can be

314 further interrupted with local ancestry from another source population upon admixture. In

315 contrast, long $\mathrm{ROH}$ are likely driven by post-admixture dynamics such as small population size

316 and mating preferences, and long $\mathrm{ROH}$ tracts may span multiple local ancestries. Indeed, we 
observed that $\mathrm{ROH}$ often span multiple ancestries, with long $\mathrm{ROH}$ spanning more switches in local ancestry per megabase. Specifically, $1.21 \%$ of shorter $\mathrm{ROH}$ contained at least one ancestry switch and $18.99 \%$ of long $\mathrm{ROH}$ contained at least one ancestry switch. Dividing ancestry switches by tract length, shorter $\mathrm{ROH}$ contain an average of 0.072 ancestry switches/Mb (standard deviation $=0.89$ ), while long $\mathrm{ROH}$ contain an average of 0.089 switches/Mb (standard deviation $=0.24)\left(\right.$ pairwise Mann-Whitney $U$ test $\left.\mathrm{p}<1 \times 10^{-8}\right)$.

While $\mathrm{ROH}$ patterns in Cabo Verde are consistent with several historical observations, we emphasize that caution is warranted in applying methods and expectations built using nonadmixed populations. We also note that, though we used a model-based $\mathrm{ROH}$ detection approach that included steps to mitigate genotyping errors and biases in allele frequency estimates, sensitivity and specificity of $\mathrm{ROH}$ detection is generally lower for shorter $\mathrm{ROH}$.

329 Further, records suggest that enslaved African individuals in Cabo Verde came from the 330 Senegambian region of Africa, and lack of genomic data from this vast region makes it difficult 331 to assess how the diversity in contributing African ethnic groups might contribute to low shorter ROH levels in Cabo Verde. These sources of uncertainty necessitate caution in inferring population history from $\mathrm{ROH}$ distributions. However, the observed differences in $\mathrm{ROH}$ among

334 the islands suggest that $\mathrm{ROH}$ are sensitive to population genetic processes even on the short 335 timescale since Cabo Verde's settlement. Together, these observations and caveats underscore 336 the need for future work testing the effects of admixture on $\mathrm{ROH}$ and on $\mathrm{ROH}$ detection, as we explore in the Discussion. 
Ancestry patterns on the $X$ chromosome vs the autosomes reflect sex-

\section{biased demographic processes}

On all islands, autosomal versus $\mathrm{X}$ chromosome ancestry patterns suggest that male and female contributions differ significantly by source population (Fig 5A). Specifically, there is higher

West African ancestry on the $\mathrm{X}$ chromosome than the autosomes (mean African ancestry proportion for the $\mathrm{X}$ chromosome $=0.76$; mean African ancestry proportion for autosomes $=$ 0.60; Wilcoxon Signed-Rank Test $\mathrm{p}<1 \times 10^{-8}$ for Santiago, Fogo, and the Northwest Cluster; $\mathrm{p}=$ 0.0013 for Boa Vista), consistent with sex-biased contributions of the founders. To quantitatively explore these differences in male vs female demographic history, we used a model-based approach to estimate differences between male and female contributions (3). A model of constant admixture is supported over a model of instantaneous admixture by historical records 
360 Given our observation that source populations make distinct contributions to $\mathrm{ROH}$ in Cabo

361 Verde, we next investigate differences in autosomal vs X chromosome ROH content. Differences

362 in autosomal vs $\mathrm{X}$ chromosome $\mathrm{ROH}$ may arise in part due to the source populations

363 contributing in a sex-biased manner. Notably, this effect would be seen most in shorter $\mathrm{ROH}$,

364 since shorter $\mathrm{ROH}$ reflect the homozygosity of older haplotypes and background relatedness

365 from the source populations. However, it is challenging to disentangle processes shaping $\mathrm{X}$ vs

366 autosomal $\mathrm{ROH}$, due to the smaller effective population size of the $\mathrm{X}$ chromosome. Our results

367 suggest that sex-biased admixture processes in Cabo Verde are reflected in $\mathrm{ROH}$, with lower

368 levels of shorter $\mathrm{ROH}$ on the $\mathrm{X}$ chromosome than on autosomes (Fig 6A). European individuals

369 have higher levels of $\mathrm{ROH}$ than West African individuals, and the higher contributions of

370 African $\mathrm{X}$ chromosomes (vs European $\mathrm{X}$ chromosomes) may drive the lower levels of short

$371 \mathrm{ROH}$ in Cabo Verde on the $\mathrm{X}$ chromosome vs the autosomes (Fig 6A). Long ROH reflects

372 different dynamics, potentially including the reduced post-admixture population size and other

373 sex-specific processes.

\section{Discussion}

375 In this study, we leveraged patterns of genetic variation in Cabo Verde to infer the demographic

376 history of the past $\sim 20$ generations. We found that distinct genetic patterns of four island

377 regions within the archipelago reflect the colonization history of the islands, including island-

378 specific settlement timing, admixture dynamics, mating patterns, and sex-biased demography. 
379 Together these results demonstrate how patterns of ancestry and genetic variation are shaped

380 by social and demographic forces on short timescales. By better understanding how complex

381 population histories generate genetic variation, we can improve interpretation of inference from

382 populations without historical records.

383 Patterns of genetic variation and admixture in Cabo Verde reflect

384 colonization history and subsequent sociocultural dynamics

385 The observed island-specific genetic patterns reflect the complex ecological and social factors

386 that shaped the settlement of Cabo Verde. Historical evidence suggests that the islands were

387 founded in a stepping-stone pattern, with settlement stages that were accompanied by

388 important economic and sociocultural shifts (35). Early written records of the population

389 (described below) suggest that admixture began early during the settlement of Cabo Verde,

390 despite the highly racially stratified, slavery-based system that characterized the first settlement

391 stage (38). The second and third stages were carried out by mostly already-admixed individuals

392 who had become a significant group within Cabo Verdean society and who migrated from the

393 southern to the northern islands (39). We found that the staggered settlement history and the

394 island-specific population dynamics shaped patterns of ancestry and genetic variation within

395 Cabo Verde.

396

397 Admixture in Cabo Verde is consistent with a model of continuous gene flow from Europe and

398 West Africa, with European males and African females contributing predominantly to the Cabo 
Verdean gene pool, as seen in other admixed populations that result from the trans-Atlantic slave trade (52,53). PCA (Supp Fig 1), IBD (Fig 1, Supp Fig 3-5), and kinship (Supp Fig 6) are consistent with genetic drift occurring during the archipelago's settlement history of consecutive founder effects and subsequent relative isolation of the islands. Specifically, individuals are genetically more related within an island than among islands, and considerably more individuals from the northern islands and Fogo are connected to each other by high IBD pairwise connections (Supp Fig 4-5). We observed differences in IBD that reflect the contributions of the source populations, such as Santiago having both the highest West African ancestry proportions and the lowest overall levels of IBD sharing. The higher African genomic ancestry on Santiago is consistent with previous results $(42,43)$, and indicates differences in settlement patterns despite the fact that colonization of the other islands involved migrations from Santiago and was based on the same slavery-based system.

Using a mechanistic model of sex-biased admixture (3), we used differences in ancestry on the $X$ chromosome vs the autosomes to examine sex-biased migration in Cabo Verde. We found that admixture occurred primarily through the mating of European males and African females, consistent with historical work that documents sex bias during the settlement of Cabo Verde. First records of the demographic distribution in Cabo Verde are found in a letter by a judicial official Pero de Guimarães to the Portuguese King in 1513, which stated the presence of 118 European individuals in Santiago, of which only four were (single) women (54). The marginal presence of European females throughout the founding of Cabo Verde contributed to the extensive genetic admixture currently found in the archipelago. The distribution of $\mathrm{ROH}$ in 
autosomes versus $\mathrm{X}$ chromosome is influenced by initial male versus female contributions to the admixed population's gene pool. Throughout the islands, the higher contributions of African X

Verde on the $X$ chromosome vs the autosomes (Fig 6A). These results underscore the long-

lasting genetic impacts of the trans-Atlantic slave trade, as has been recently shown in admixed populations across the Americas (53).

Despite only a few hundred years of unique population histories among the islands, we also suggesting ancestry-assortative mating on the short timescale since the founding of the islands. 
enslavers sexually coerced enslaved African women during slavery. After the end of slavery,

racially unbalanced control of landownership and higher social status of Europeans resulted in structure can impact population homozygosity and differentiation. was settled shortly after Santiago. Fogo's society was, since its origins, a conservative rural society whose main economic activity was to produce goods to trade in the African coast. The socioeconomic elite was patriarchal and aristocratic (40), and composed primarily of related

462 families, which promoted first-cousin marriages (41). The sustenance of this class depended on

463 land ownership, which allowed the slavery-based system to be perpetuated longer in Fogo than 464 in Santiago $(35,40)$. The unique attributes of Fogo are reflected in population genetic patterns. 
465 For example, we found that African ancestry proportion align with kinship patterns overall, but

466 exceptions were most obvious in individuals from Fogo and the Northwest Cluster, where some

467 observed relatedness patterns are not as clearly explained by global ancestry proportions. In our

468 estimation of admixture timing using local ancestry disequilibrium, we observed that Fogo has

469 greater levels of local ancestry disequilibrium than the other islands. Higher local ancestry

470 disequilibrium may be driven by ancestry-assortative mating. Additionally, it may be that Fogo

471 has experienced stronger founder effects, which would decrease the number of ancestral

472 lineages. Indeed, the higher levels of shorter $\mathrm{ROH}$ within Fogo (Fig 4; Supp Fig 9) are consistent

473 with founder effects increasing background relatedness and thus increasing shorter $\mathrm{ROH}$.

474 Though we emphasize the need for further work to understand the dynamics of $\mathrm{ROH}$ in

475 admixed populations, these patterns are in agreement with the lower Y-chromosome haplotype

476 diversity observed in Fogo compared to the other islands (42). Long ROH and IBD patterns

477 gives us further insights about the post-admixture demographic processes in the archipelago,

478 including uncovering genetic consequences of consecutive founder effects.

479 Patterns of genetic variation and admixture in Cabo Verde influence

480 demographic inference

481 The admixture dynamics, founder effects, and mating patterns within Cabo Verde shape

482 summaries of genetic variation that are often used to inform demographic inference, such as

483 local ancestry disequilibrium, IBD, and $\mathrm{ROH}$. To investigate the effects of these population

484 dynamics on demographic inference, we estimated admixture timing using multiple population 
485

486

487

488

489

490

491

492

493

494

495

496

497

498

499

500

501

502

503

504

505

genetic tools. Estimates of admixture timing from genetic data were most concordant with historical records when using inference based on local ancestry disequilibrium (7), allowing for ancestry-assortative mating and migration after the founding of the admixed population. Under this method, we tested a range of ancestry-assortative mating strengths ( 0 vs values inferred using ANCESTOR in Fig 3 and a broader range of values in Supp Fig 8) and migration rates (0 vs $1 \%$ each generation). The migration levels are used to demonstrate the trends of timing estimates using constant migration and assortative mating, rather than to obtain a specific estimate of the migration parameters. Consistent with previous theoretical work on the impact of assortative mating on the timing of admixture, we find that estimates that do not account for ancestry assortment are more recent than historical records and than estimates that include ancestry assortment $(7,58)$. Under random mating, haplotypes are distributed randomly in mating pairs in a population, allowing recombination to shuffle haplotypes over generations. In contrast, ancestry-assortative mating can keep ancestral haplotypes more intact, leading to underestimation of the number of generations of admixture when estimating admixture timing. Zaitlen et al. (2017) and Goldberg et al. (2020) theoretically demonstrate that certain models of non-random mating in admixed populations maintain variation in the ancestry proportion and linkage over time-summary statistics that will make admixture appear more recent than it is when mating patterns are not considered $(7,58)$. Our evidence of ancestry-assortative mating in Cabo Verde underscores the importance of accounting for nonrandom mating in understanding admixture and inferring demographic history. Assortative mating has been documented with respect to genetic ancestry, socioeconomic factors, and phenotypic characteristics $(7,13-15,59)$, 
506 but remains an under-recognized force in human population genetics and demographic

507 inference.

508

509 Notably, assortative mating alone was not enough to obtain time of admixture estimates

510 concordant with historical records. Admixture timing estimates were most concordant with the

511 historical records when allowing both assortative mating and constant admixture. An

512 admixture scenario that allows multi-wave or constant admixture model is compatible with

513 historical work suggesting constant migration from both Europe and West African throughout

514 the settlement of the islands. Importantly, the authors of ALDER and MultiWaver point out that

515 methods for inferring admixture timing have varying sensitivities to different admixture

516 scenarios $(4,5)$. MultiWaver may not be able to accurately infer demographic histories that

517 deviate from its pre-defined admixture models. ALDER notes that multi-wave or continuous

518 admixture can lead to more recent estimates of admixture timing, as the method inherently

519 assumes single-point admixture. Despite this caveat, ALDER is frequently applied in cases

520 where admixture is not strictly instantaneous.

522 All of the timing methods we used placed admixture timing for the different islands closer

523 together than historical dates of settlement, consistent with historical expectations that the

524 initial admixture in the southern islands was significant, and that many individuals that

525 occupied the northern and eastern islands during the second and third settlement stages of

526 Cabo Verde were already admixed. For example, the serial founding of the islands may explain

527 why estimates of admixture timing for Boa Vista were closer to historical records. While Boa 
Vista was founded most recently, it was founded by already admixed individuals. This observation, together with IBD and kinship patterns, support the serial founding of the groups of islands as the main model of settlement of Cabo Verde, as opposed to their independent settling as some historical data suggest (35). This type of serial founding scenario is common throughout recent human migration, underscoring that settlement patterns, in addition to settlement timing, are critical components of accurately inferring human demographic history.

We found that Cabo Verde's island-specific demographic history and admixture dynamics have important genomic consequences, as observed with $\mathrm{ROH}$. Despite the relatively recent colonization of the islands, some individuals presented even lower overall levels of $\mathrm{ROH}$ than African reference populations. We found that low overall levels of $\mathrm{ROH}$ in Cabo Verde are driven by shorter length $\mathrm{ROH}$. This observation is consistent with the idea that shorter $\mathrm{ROH}$ can be attributed to older shared ancestors from the source populations, and these tracts can be interrupted with admixed ancestry upon admixture. However, many admixed individuals still present excess long $\mathrm{ROH}$, likely reflecting post-admixture processes such as serial founding and ancestry-assortative mating. The observed island-specific distributions of $\mathrm{ROH}$ are consistent with the colonization history of the islands. For example, the cultural dynamics and higher rates of first-cousin marriages in Fogo (41), possibly driving high levels of long ROH (Fig 4B). In contrast, Santiago has both the oldest population and the largest population size, and has comparatively low levels of both shorter and long $\mathrm{ROH}$. These observations suggest that more work, both empirical and theoretical, is needed to understand the interacting forces of local ancestry and $\mathrm{ROH}$. 
551 In sum, we provide insights into the population history of Cabo Verde and demonstrate how

552 admixed populations can provide powerful test cases for understanding demographic processes

553 and genomic consequences in recent human history. We show that patterns of shared ancestry

554 between and within the islands (quantified with IBD and kinship estimates) reflect serial

555 founder effects as well as settlement patterns such as post-admixture nonrandom mating. We

556 find that accounting for nonrandom mating allows us to improve inference of admixture timing

557 and better contextualize genomic consequences of admixture dynamics, such as $\mathrm{ROH}$. We find

558 that differences in ancestry on the $\mathrm{X}$ chromosome vs the autosomes reflect sex-biased

559 demographic processes. Given the ubiquity of admixture throughout modern human

560 population, these results provide important, generalizable considerations for the study of recent

561 human evolution.

\section{Methods}

\section{Study population and ancestry reference panels}

564 We used genotype data from Beleza et al. (2013) (43), which included 563 Cabo Verdeans from

565 the following regions of the archipelago: Santiago $(n=172)$, Fogo $(n=129)$, Boa Vista $(n=26)$,

566 and the three northwestern islands in aggregate (Northwest Cluster; $n=236$ ) (Figure 1; Supp

567 Fig 1). The genotype data exclude cryptic related individuals (first-degree relatives) identified

568 by kinship analyses at the time of sampling. In accordance with historical records and previous 
work showing that Iberian and Senegambian populations are suitable proxies for the ancestry sources of Cabo Verde (45), we leveraged data from the 1000 Genomes Project to estimate admixture proportions and call local ancestry in the Cabo Verde individuals, as described below. We merged the Cabo Verde genotypes with genotypes from 107 GWD (Gambian in Western Division - Mandinka) and 107 IBS (Iberian Population in Spain) samples called from high-coverage resequencing data released through the International Genome Sample Resource $(60,61)$. Our final merged dataset consisted of 884,656 autosomal and 20,967 X chromosome SNPs shared between the Cabo Verde samples and the reference samples, with average missingness rates of 0.0017 by SNP for autosomes, and 0.0024 for the $\mathrm{X}$ chromosome.

\section{Historical records}

Throughout our analyses, we draw comparisons between genetic results and historical records.

We used primary historical documents, mainly historical letters to the Portuguese Crown, documenting demographic characteristics of the islands across time. Our analyses of the historical data took into consideration the generalized interpretations of historian scholars such as Correia e Silva $(2001 ; 2002)(35,39)$, Cabral $(2001 ; 2012)(38,40)$ and Baleno $(2001)(36)$. These sources document the dates of the stages of settlement mentioned above, along with associated with changes in the economy. We refer to the interpretation of genealogical data by Cabral (2012) (40) and Barbosa (1997) (41) for evidence of the structure of the Fogo's society and for evidence of first-cousin marriages within Fogo (41). 


\section{Characterization of ancestry}

589

590

591

592

593

594

595

596

597

598

599

600

601

602

603

604

605

606

607

608

To produce estimates of admixture proportions, we first performed unsupervised clustering of the samples using ADMIXTURE v1.3.0 (62) (see Supp Table 3 for a summary of all computational methods used in this study). ADMIXTURE was run separately for the autosomal and $\mathrm{X}$ chromosome datasets after pruning based on linkage disequilibrium (LD) using the indep-pairwise option of PLINK v1.9 with a 50-SNP sliding window incremented by 10 SNPs, and an LD threshold of r2 = 0.5 (63). This pruned dataset included 514,551 autosomal and 12,706 X chromosome SNPs. We estimated individual ancestries by averaging over ten independent unsupervised ADMIXTURE runs using $\mathrm{K}=2$, given the historical and genetic support for two source populations $(43,45)$. Using the LD-pruned dataset, we also visualized the data using PLINK's principal component analysis (--pca) function, confirming that the samples cluster approximately based on their geographic memberships, and that West African vs European ancestry clearly separates on the first principal component (Supp Fig 1). We phased all samples using SHAPEIT2 (64). After running SHAPEIT -check to exclude sites not contained within the reference map, we ran SHAPEIT to yield phased genotypes at 881,279 autosomal SNPs and 20,793 X chromosome SNPs. We then called local ancestry with RFMix v1.5.4 (65) PopPhased under a two-way admixture model using the West African and European reference genotypes described above. These local ancestry calls are publicly available via Zenodo (66). Ancestry proportions estimated with ADMIXTURE and RFMix here are highly correlated with results from Beleza et al. (2013) (43), using the same genotype data but with different software (frappe (67) and SABER (68)) and an older reference dataset (Supp Fig 2). To test whether phasing and 
609 local ancestry calls were robust against the choice of reference panels, we repeated the

610 SHAPEIT and RFMix steps using a reference dataset composed by all African (ACB, ASW, ESN,

611 GWD, LWK, MSL, and YRI) and European (CEU, FIN, GBR, IBS, and TSI) populations available

612 from the high-coverage resequencing data released by the 1000 Genomes Project $(60,61)$. The

613 resulting local ancestry calls with all AFR and EUR reference panels correlated closely with calls

614 using GWD and IBS as reference panels (Supp Fig 2C). We also performed local ancestry calling

615 using ELAI, a method that performs both phasing and local ancestry assignment (69). Again

616 using the IBS and GWD reference genotypes described above, we ran ELAI under a two-way

617 admixture model using the following parameters: -mg (number of generations) 20, -s (EM steps)

$61830,-\mathrm{C}$ (upper clusters) 2, and -c (lower clusters) 10. The resulting local ancestry calls from ELAI

619 correlated closely with calls from RFMix (Supp Fig 2D).

\section{Inference of admixture timing}

621 We applied three distinct strategies for estimating the timing of the onset of admixture in Cabo

622 Verde: ALDER (4), MultiWaver 2.0 (5), and a method based on patterns of linkage

623 disequilibrium between local ancestry tracts (7). We first converted the genotypes to 
630

631

632

633

634

635

636

637

638

639

640

641

642

643

644

645

646

647

with the first SNP on each autosome, used the $10 \mathrm{Mb}$ window end point to identify the SNP

closest to the inside of this boundary, and then used local ancestry calls at these positions to

determine LAD. We repeated this process along each chromosome to obtain LAD in 279

autosomal windows. Using possible values of admixture generations ranging from 5-25, we

determined the best fit using island-specific mean LAD decay over the 279 autosomal windows,

assortative mating parameters estimated with ANCESTOR (see below), a starting autosomal

admixture proportion of 0.65 , and either no migration or with migration (migration rate $=0.01$ ).

\section{Tests for ancestry-assortative mating}

We tested for ancestry-assortative mating over the last generation using $\operatorname{ANCESTOR}(15,72)$.

ANCESTOR uses phased local ancestry tracts in the current generation to estimate the ancestral proportions of the two parents of each individual. We used the inferred parental ancestries to

test for assortative mating seen as a positive correlation in ancestry between inferred mating pairs, and we used the strength of the observed positive correlation (Pearson's R for each population, using the median value from all chromosomes) as the assortative mating parameter in our application of the LAD-based method for inferring admixture timing. We also examined evidence of nonrandom mating by estimating genetic relatedness within Cabo Verde using both IBD (see below) and kinship coefficients (46).

\section{Identification of IBD tracts and $\mathrm{ROH}$}

Following the analysis pipeline of S. R. Browning et al. (2018) (2), we inferred segments of IBD using the haplotype-based IBD detection method Refined IBD (73), and we estimated ancestry- 
650

651

652

653

654

655

656

657

658

659

660

661

662

663

664

665

666

667

668

669

670

671

specific population size using IBDNe (1). After running Refined IBD, we used its gap-filling utility to remove gaps between segments less than $0.6 \mathrm{cM}$ that had at most one discordant homozygote. We then filtered out IBD segments smaller than $5 \mathrm{cM}$, as short segments below this threshold are difficult to accurately detect (74). To visualize IBD sharing in Fig 1 and Supp

Fig 3, the sum of IBD shared in each pairwise comparison of individuals was plotted by using Cytoscape 3.8 to group nodes by island, position nodes within each island according to a prefuse force-directed algorithm, and scale the color of edges based on log-transformed total IBD length (75).

We called ROH using GARLIC v.1.1.6 (49), which implements the ROH calling pipeline of Pemberton et al. (2012) (30). We performed this analysis separately for the autosomes and X chromosome. Based on the pipeline described in Szpiech et al. (2017), we used a single constant genotyping error rate of 0.001 , we allowed GARLIC to automatically choose a window size for each population (--auto-winsize), and we used the resample flag to mitigate biases in allele frequency estimates caused by differing sample sizes. This resulted in GARLIC selecting a window size of 50 SNPs in each of the Cabo Verde regions and in GWD, and a window size of 40 for IBS. Using a three-component Gaussian mixture model, GARLIC classified ROH into three length groups: small/class A, medium/class B, and long/class C ROH. Across all populations, class $\mathrm{A} / \mathrm{B}$ and class $\mathrm{B} / \mathrm{C}$ size boundaries were inferred as approximately $300 \mathrm{~kb}$ and $1 \mathrm{Mb}$, respectively (see Supp Table 2 for population-specific parameters, including LOD cutoffs and size boundaries). Using only the females, we then classified $\mathrm{ROH}$ for the $\mathrm{X}$ chromosome. 
672 To evaluate the influence of $\mathrm{ROH}$ size classification cutoffs in the results, we repeated size

673 classification using the --size-bounds flag in GARLIC to impose four additional sets of size class

674 boundaries. We first collected the population-specific length classification cut-offs from the 64

675 worldwide populations provided by Pemberton et al. (2012), which represents an extensive

676 range of classification schemes. We repeated ROH-calling with GARLIC using the minimum,

677 mean, and maximum of the 64 population-specific boundaries reported in Pemberton et al.

678 (2012) (Supp Fig 9A-C). Finally, we classified ROH based on the commonly used rule of thumb

679 that a time depth of $m$ meioses is expected to give a mean IBD segment length of $100 / m \mathrm{cM}(76)$.

680 In this case, common ancestors within the past 20 generations (post-admixture) would give an

681 expected IBD segment length of approximately $2.5 \mathrm{Mb}$ or longer, so we applied $2.5 \mathrm{Mb}$ as the

682 shorter/long ROH boundary in Supp Fig 9D.

\section{Estimation of sex bias}

684 To examine sex bias, we applied a mechanistic model of sex-biased admixture (3) to infer

685 admixture parameters under a model of constant migration. We performed this analysis for all

686563 Cabo Verdean individuals pooled together, and then repeated the analysis for each island

687 independently. We provided the model with the following parameters: grid increment size

688 (0.02), the observed autosomal and X admixture proportions (based on ADMIXTURE estimates

689 described above), the proportion of females in the sample, and the percentile cutoff for

690 parameters to keep $(0.1 \%)$. 


\section{Acknowledgements}

692 We thank Jorge Rocha for his contribution during project conception and sampling, and Greg

693 Barsh for his support in later stages of data collection. We thank Noah Zaitlen for sharing

694 scripts to assist in our application of the Zaitlen et al. (2017) local ancestry disequilibrium

695 method. This work was supported by MRC-UK MR/M01987X/1 and FCT-Portugal PTDC/BIA-

696 BDE/64044/2006 awarded to Sandra Beleza; NIH NIGMS grant R35 GM133481 awarded to

697 Amy Goldberg; NIH NIGMS grant F32 GM139313 awarded to Katharine Korunes; and by

698 CNPq (CSF/PDE 207651/2014-0) awarded to Giordano Bruno Soares-Souza. We acknowledge

699 the source of the reference datasets: 1000G 30x data generated at the New York Genome Center

700 with funds provided by NHGRI Grant 3UM1HG008901-03S1. We thank Dr. Kirk Lohmueller

701 and three anonymous reviewers for their helpful feedback on this work. Finally, we would like

702 to thank the Cabo Verdean participants for their invaluable contributions, and the University of

703 Cabo Verde administration for their support.

704 Declaration of interests

705 The authors declare no competing interests.

706 Data and Code Availability

707 Sampling consent forms do not allow for public release of genotype data. Inferred local ancestry

708 information can be found at https://doi.org/10.5281/zenodo.4021277. Code generated for this

709 study can be found at https://github.com/agoldberglab/CaboVerde_Demographic_Analyses. 


\section{References}

711

712

713

714

715

716

717

718

719

720

721

722

723

724

725

726

727

728

729

730

731

732

733

734

735

736

737

738

739

740

741

742

1. Browning SR, Browning BL. Accurate Non-parametric Estimation of Recent Effective Population Size from Segments of Identity by Descent. Am J Hum Genet. 2015 Sep 3;97(3):404-18.

2. Browning SR, Browning BL, Daviglus ML, Durazo-Arvizu RA, Schneiderman N, Kaplan $\mathrm{RC}$, et al. Ancestry-specific recent effective population size in the Americas. PLOS Genet. 2018 May 24;14(5):e1007385.

3. Goldberg A, Rosenberg NA. Beyond 2/3 and 1/3: The Complex Signatures of Sex-Biased Admixture on the X Chromosome. Genetics. 2015 Sep;201(1):263-79.

4. Loh P-R, Lipson M, Patterson N, Moorjani P, Pickrell JK, Reich D, et al. Inferring admixture histories of human populations using linkage disequilibrium. Genetics. 2013 Apr;193(4):1233-54.

5. Ni X, Yuan K, Liu C, Feng Q, Tian L, Ma Z, et al. MultiWaver 2.0 : modeling discrete and continuous gene flow to reconstruct complex population admixtures. Eur J Hum Genet. 2019 Jan;27(1):133-9.

6. Schiffels S, Durbin R. Inferring human population size and separation history from multiple genome sequences. Nat Genet. 2014 Aug;46(8):919-25.

7. Zaitlen N, Huntsman S, Hu D, Spear M, Eng C, Oh SS, et al. The Effects of Migration and Assortative Mating on Admixture Linkage Disequilibrium. Genetics. 2017 Jan;205(1):37583.

8. Busby GBJ, Hellenthal G, Montinaro F, Tofanelli S, Bulayeva K, Rudan I, et al. The Role of Recent Admixture in Forming the Contemporary West Eurasian Genomic Landscape. Curr Biol. 2015 Oct 5;25(19):2518-26.

9. Fernandes V, Brucato N, Ferreira JC, Pedro N, Cavadas B, Ricaut F-X, et al. Genome-Wide Characterization of Arabian Peninsula Populations: Shedding Light on the History of a Fundamental Bridge between Continents. Mulligan C, editor. Mol Biol Evol. 2019 Mar;36(3):575-86.

10. Font-Porterias N, Arauna LR, Poveda A, Bianco E, Rebato E, Prata MJ, et al. European Roma groups show complex West Eurasian admixture footprints and a common South Asian genetic origin. PLOS Genet. 2019 Sep 23;15(9):e1008417.

11. Lazaridis I, Patterson N, Mittnik A, Renaud G, Mallick S, Kirsanow K, et al. Ancient human genomes suggest three ancestral populations for present-day Europeans. Nature. 2014 Sep 18;513(7518):409-13. 
12. Skoglund $\mathrm{P}$, Malmström $\mathrm{H}$, Raghavan $\mathrm{M}$, Storå J, Hall $\mathrm{P}$, Willerslev E, et al. Origins and genetic legacy of Neolithic farmers and hunter-gatherers in Europe. Science. $2012 \mathrm{Apr}$ 27;336(6080):466-9.

13. Risch N, Choudhry S, Via M, Basu A, Sebro R, Eng C, et al. Ancestry-related assortative mating in Latino populations. Genome Biol. 2009 Nov 20;10(11):R132.

14. Sebro R, Peloso GM, Dupuis J, Risch NJ. Structured mating: Patterns and implications. PLOS Genet. 2017 Apr 6;13(4):e1006655.

15. Zou JY, Park DS, Burchard EG, Torgerson DG, Pino-Yanes M, Song YS, et al. Genetic and socioeconomic study of mate choice in Latinos reveals novel assortment patterns. Proc Natl Acad Sci U S A. 2015 Nov;112(44):13621-6.

16. Arbiza L, Gottipati S, Siepel A, Keinan A. Contrasting X-Linked and Autosomal Diversity across 14 Human Populations. Am J Hum Genet. 2014 Jun 5;94(6):827-44.

17. Bustamante CD, Ramachandran S. Evaluating signatures of sex-specific processes in the human genome. Nat Genet. 2009 Jan;41(1):8-10.

18. Keinan A, Mullikin JC, Patterson N, Reich D. Accelerated genetic drift on chromosome X during the human dispersal out of Africa. Nat Genet. 2009 Jan;41(1):66-70.

19. Ramachandran S, Rosenberg NA, Feldman MW, Wakeley J. Population differentiation and migration: Coalescence times in a two-sex island model for autosomal and X-linked loci. Theor Popul Biol. 2008 Dec 1;74(4):291-301.

20. Wilkins JF, Marlowe FW. Sex-biased migration in humans: what should we expect from genetic data? BioEssays. 2006;28(3):290-300.

21. Busby GB, Band G, Si Le Q, Jallow M, Bougama E, Mangano VD, et al. Admixture into and within sub-Saharan Africa. Pickrell JK, editor. eLife. 2016 Jun 21;5:e15266.

22. Hellenthal G, Busby GBJ, Band G, Wilson JF, Capelli C, Falush D, et al. A Genetic Atlas of Human Admixture History. Science. 2014 Feb 14;343(6172):747-51.

23. Laso-Jadart R, Harmant C, Quach H, Zidane N, Tyler-Smith C, Mehdi Q, et al. The Genetic Legacy of the Indian Ocean Slave Trade: Recent Admixture and Post-admixture Selection in the Makranis of Pakistan. Am J Hum Genet. 2017 Dec;101(6):977-84.

24. Ruiz-Linares A, Adhikari K, Acuña-Alonzo V, Quinto-Sanchez M, Jaramillo C, Arias W, et al. Admixture in Latin America: Geographic Structure, Phenotypic Diversity and SelfPerception of Ancestry Based on 7,342 Individuals. PLOS Genet. 2014 Sep 25;10(9):e1004572. 
25. Triska P, Soares P, Patin E, Fernandes V, Cerny V, Pereira L. Extensive Admixture and Selective Pressure Across the Sahel Belt. Genome Biol Evol. 2015 Dec 1;7(12):3484-95.

26. Korunes KL, Goldberg A. Human genetic admixture. PLOS Genet. 2021 Mar 11;17(3):e1009374.

27. Blant A, Kwong M, Szpiech ZA, Pemberton TJ. Weighted likelihood inference of genomic autozygosity patterns in dense genotype data. BMC Genomics. 2017 Dec 1;18(1):928.

28. Kirin M, McQuillan R, Franklin CS, Campbell H, McKeigue PM, Wilson JF. Genomic Runs of Homozygosity Record Population History and Consanguinity. PLOS ONE. 2010 Nov 15;5(11):e13996.

29. McQuillan R, Leutenegger A-L, Abdel-Rahman R, Franklin CS, Pericic M, Barac-Lauc L, et al. Runs of Homozygosity in European Populations. Am J Hum Genet. 2008 Sep 12;83(3):359-72.

30. Pemberton TJ, Absher D, Feldman MW, Myers RM, Rosenberg NA, Li JZ. Genomic Patterns of Homozygosity in Worldwide Human Populations. Am J Hum Genet. 2012 Aug;91(2):275-92.

31. Mooney JA, Huber CD, Service S, Sul JH, Marsden CD, Zhang Z, et al. Understanding the Hidden Complexity of Latin American Population Isolates. Am J Hum Genet. 2018 Nov 1;103(5):707-26.

32. Popejoy AB, Fullerton SM. Genomics is failing on diversity. Nature. 2016 13;538(7624):1614.

33. Popejoy AB, Ritter DI, Crooks K, Currey E, Fullerton SM, Hindorff LA, et al. The clinical imperative for inclusivity: Race, ethnicity, and ancestry (REA) in genomics. Hum Mutat. 2018;39(11):1713-20.

34. Landry LG, Ali N, Williams DR, Rehm HL, Bonham VL. Lack Of Diversity In Genomic Databases Is A Barrier To Translating Precision Medicine Research Into Practice. Health Aff Proj Hope. 2018;37(5):780-5.

35. Correia e Silva AL. Dinâmicas de decomposição e recomposição de espaços e sociedades. In: Santos, MEM, editor. História geral de Cabo Verde. Lisbon, Portugal; Praia, Cabo Verde: Instituto de Investigação Científica Tropical, Instituto Nacional de Investigação, Promoção e Património Culturais de Cabo Verde; 2002. p. 1-66.

36. Baleno IC. Povoamento e Formação da Sociedade. In: Albuquerque, L and Santos, MEM, editor. História geral de Cabo Verde. Lisbon, Portugal; Praia, Cabo Verde: Instituto de Investigação Científica Tropical, Instituto Nacional de Investigação, Promoção e Património Culturais de Cabo Verde; 2001. p. 125-77. 
37. Russell-Wood A. The Portuguese Empire, 1415-1808: A World on the Move. Baltimore, Maryland: Johns Hopkins University Press; 1998. 289 p.

38. Cabral I. Ribeira Grande: vida urbana, gente, mercancia, estagnação. In: Santos, MEM, editor. História geral de Cabo Verde. Lisbon, Portugal; Praia, Cabo Verde: Instituto de Investigação Científica Tropical, Instituto Nacional de Investigação, Promoção e Património Culturais de Cabo Verde; 2001. p. 225-73.

39. Correia e Silva AL. Espaço, ecologia e economia interna. In: de Albuquerque L, Santos, MEM, editor. História geral de Cabo Verde. Lisbon, Portugal; Praia, Cabo Verde: Instituto de Investigação Científica Tropical, Instituto Nacional de Investigação, Promoção e Património Culturais de Cabo Verde; 2001.

40. Cabral I. A evolução da sociedade foguense: Através de um estudo prosopográfico da elite da ilha (sec. XVI-XVIII). 2012.

41. Barbosa, Gilda. Casamento entre primos. J Terra Nova No 246. 1997;

42. Beleza S, Campos J, Lopes J, Araújo II, Hoppfer Almada A, e Silva AC, et al. The Admixture Structure and Genetic Variation of the Archipelago of Cape Verde and Its Implications for Admixture Mapping Studies. Relethford JH, editor. PLoS ONE. 2012 Nov;7(11):e51103.

43. Beleza S, Johnson NA, Candille SI, Absher DM, Coram MA, Lopes J, et al. Genetic Architecture of Skin and Eye Color in an African-European Admixed Population. Spritz RA, editor. PLoS Genet. 2013 Mar;9(3):e1003372.

44. Fernandes AT, Velosa R, Jesus J, Carracedo A, Brehm A. Genetic differentiation of the Cabo Verde archipelago population analysed by STR polymorphisms. Ann Hum Genet. 2003 Jul;67(Pt 4):340-7.

45. Verdu P, Jewett EM, Pemberton TJ, Rosenberg NA, Baptista M. Parallel Trajectories of Genetic and Linguistic Admixture in a Genetically Admixed Creole Population. Curr Biol. 2017;27(16):2529-2535.e3.

46. Ochoa A, Storey JD. New kinship and FST estimates reveal higher levels of differentiation in the global human population. bioRxiv. 2019 May;653279.

47. Reed FA, Tishkoff SA. African human diversity, origins and migrations. Curr Opin Genet Dev. 2006 Dec 1;16(6):597-605.

48. Tishkoff SA, Williams SM. Genetic analysis of African populations: human evolution and complex disease. Nat Rev Genet. 2002 Aug;3(8):611-21. 
49. Szpiech ZA, Blant A, Pemberton TJ. GARLIC: Genomic Autozygosity Regions Likelihoodbased Inference and Classification. Berger B, editor. Bioinformatics. 2017 Jul;33(13):2059_ 62.

50. Ceballos FC, Joshi PK, Clark DW, Ramsay M, Wilson JF. Runs of homozygosity: windows into population history and trait architecture. Nat Rev Genet. 2018 Apr;19(4):220-34.

51. Goldberg A, Günther T, Rosenberg NA, Jakobsson M. Ancient X chromosomes reveal contrasting sex bias in Neolithic and Bronze Age Eurasian migrations. Proc Natl Acad Sci. 2017 Mar 7;114(10):2657-62.

52. Eltis D, Richardson D, Blight DW. Atlas of the Transatlantic Slave Trade [Internet]. Yale University Press; 2010 [cited 2020 Oct 9]. Available from: https://www.jstor.org/stable/j.ctt5vm1s4

53. Micheletti SJ, Bryc K, Esselmann SGA, Freyman WA, Moreno ME, Poznik GD, et al. Genetic Consequences of the Transatlantic Slave Trade in the Americas. Am J Hum Genet. 2020 Aug 6;107(2):265-77.

54. Pero de Guimarães. Corregedor de Cabo Verde, carta de 22 de Maio de 1513. In: de Albuquerque L, Santos, MEM, editor. História geral de Cabo Verde. Lisbon, Portugal; Praia, Cabo Verde: Instituto de Investigação Científica Tropical, Instituto Nacional de Investigação, Promoção e Património Culturais de Cabo Verde; 1991. p. 219-23.

55. Moreno-Estrada A, Gravel S, Zakharia F, McCauley JL, Byrnes JK, Gignoux CR, et al. Reconstructing the Population Genetic History of the Caribbean. PLOS Genet. 2013 Nov 14;9(11):e1003925.

56. Campbell CL, Palamara PF, Dubrovsky M, Botigué LR, Fellous M, Atzmon G, et al. North African Jewish and non-Jewish populations form distinctive, orthogonal clusters. Proc Natl Acad Sci [Internet]. 2012 Jul 31 [cited 2021 Aug 2]; Available from: https://www.pnas.org/content/early/2012/07/31/1204840109

57. Seibert G. Crioulização em Cabo Verde e São Tomé e Príncipe: divergências históricas e identitárias. Afro-Ásia. 2014 Jun 1;41-70.

58. Goldberg A, Rastogi A, Rosenberg NA. Assortative mating by population of origin in a mechanistic model of admixture. Theor Popul Biol. 2020 Aug 1;134:129-46.

59. Robinson MR, Kleinman A, Graff M, Vinkhuyzen AAE, Couper D, Miller MB, et al. Genetic evidence of assortative mating in humans. Nat Hum Behav. 2017 Jan 9;1(1):1-13.

60. Clarke L, Fairley S, Zheng-Bradley X, Streeter I, Perry E, Lowy E, et al. The international Genome sample resource (IGSR): A worldwide collection of genome variation incorporating the 1000 Genomes Project data. Nucleic Acids Res. 2017 Jan 4;45(D1):D854-9. 
61. Fairley S, Lowy-Gallego E, Perry E, Flicek P. The International Genome Sample Resource (IGSR) collection of open human genomic variation resources. Nucleic Acids Res. 2020 Jan 8;48(D1):D941-7.

62. Alexander DH, Novembre J, Lange K. Fast model-based estimation of ancestry in unrelated individuals. Genome Res. 2009 Sep;19(9):1655-64.

63. Purcell S, Neale B, Todd-Brown K, Thomas L, Ferreira MAR, Bender D, et al. PLINK: a tool set for whole-genome association and population-based linkage analyses. Am J Hum Genet. 2007 Sep;81(3):559-75.

64. Delaneau O, Zagury J-F, Marchini J. Improved whole-chromosome phasing for disease and population genetic studies. Nat Methods. 2013 Jan;10(1):5-6.

65. Maples BK, Gravel S, Kenny EE, Bustamante CD. RFMix: a discriminative modeling approach for rapid and robust local-ancestry inference. Am J Hum Genet. 2013 Aug;93(2):278-88.

66. Hamid I, Korunes K, Beleza S, Goldberg A. Rapid adaptation to malaria facilitated by admixture in the human population of Cabo Verde [Internet]. Zenodo; 2020 [cited 2020 Sep 21]. Available from: https://zenodo.org/record/4021277\#.X2kMuPxKhTY

67. Tang H, Peng J, Wang P, Risch NJ. Estimation of individual admixture: analytical and study design considerations. Genet Epidemiol. 2005 May;28(4):289-301.

68. Tang H, Coram M, Wang P, Zhu X, Risch N. Reconstructing Genetic Ancestry Blocks in Admixed Individuals. Am J Hum Genet. 2006 Jul;79(1):1-12.

69. Guan Y. Detecting structure of haplotypes and local ancestry. Genetics. 2014 Mar;196(3):625-42.

70. Patterson N, Price AL, Reich D. Population Structure and Eigenanalysis. PLOS Genet. 2006 Dec 22;2(12):e190.

71. Price AL, Patterson NJ, Plenge RM, Weinblatt ME, Shadick NA, Reich D. Principal components analysis corrects for stratification in genome-wide association studies. Nat Genet. 2006 Aug;38(8):904-9.

72. Zou JY, Halperin E, Burchard E, Sankararaman S. Inferring parental genomic ancestries using pooled semi-Markov processes. Bioinformatics. 2015 Jun 15;31(12):i190-6.

73. Browning BL, Browning SR. Improving the accuracy and efficiency of identity-by-descent detection in population data. Genetics. 2013 Jun;194(2):459-71. 
906

907

908

909

910

911

912

913

914
74. Nelson D, Kelleher J, Ragsdale AP, Moreau C, McVean G, Gravel S. Accounting for longrange correlations in genome-wide simulations of large cohorts. PLOS Genet. 2020 May 5;16(5):e1008619.

75. Shannon P, Markiel A, Ozier O, Baliga NS, Wang JT, Ramage D, et al. Cytoscape: A Software Environment for Integrated Models of Biomolecular Interaction Networks. Genome Res. 2003 Nov;13(11):2498-504.

76. Thompson EA. Identity by Descent: Variation in Meiosis, Across Genomes, and in Populations. Genetics. 2013 Jun;194(2):301-26. 


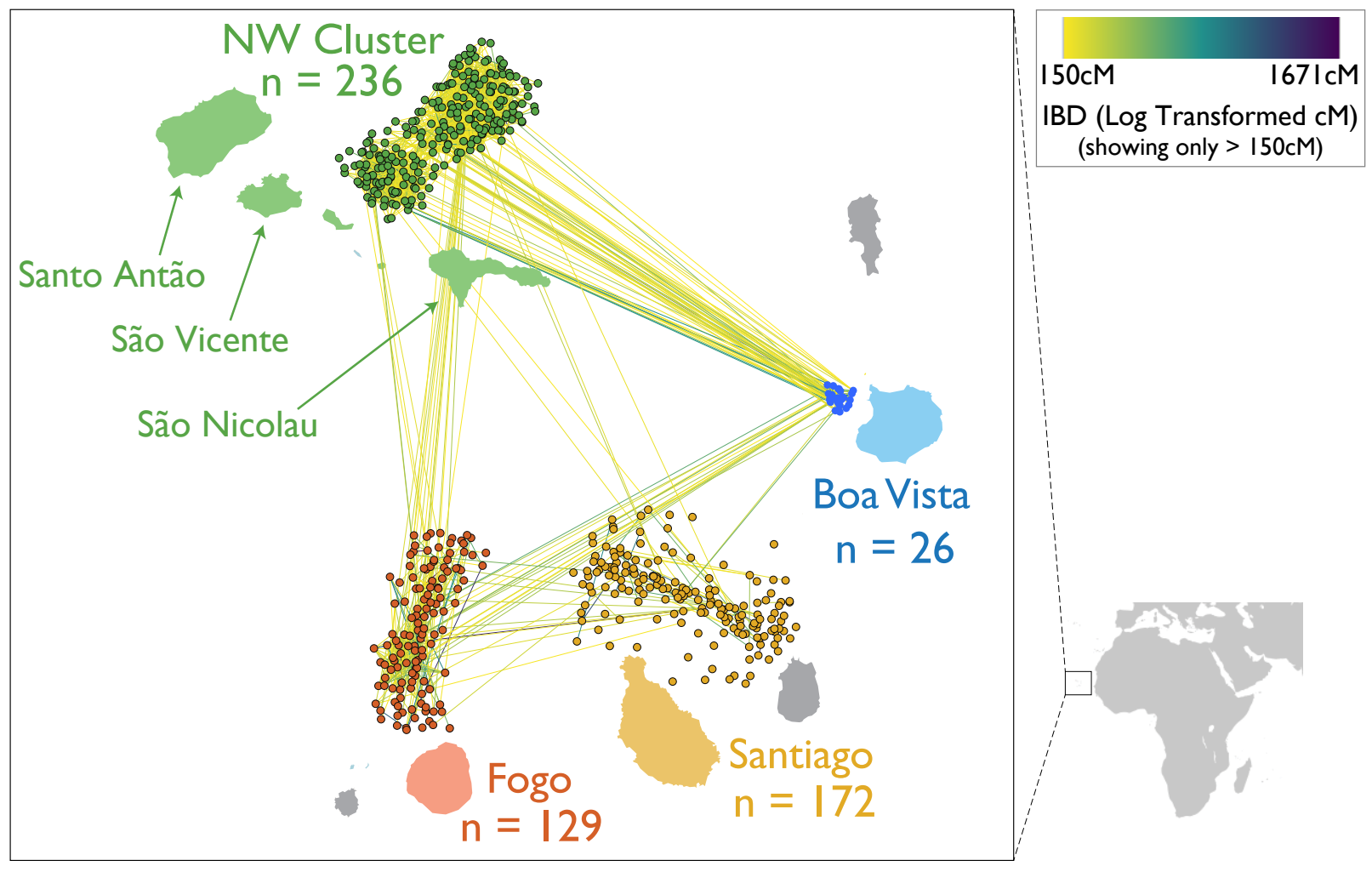

Fig 1 I Shared ancestry between and within the islands in the context of geography. Each island has a corresponding cluster of nodes representing all sampled individuals, with the individuals localized to be adjacent to the island where they were sampled. Node placement within islands is determined by a force-directed algorithm using pairwise shared IBD, meaning that the spread of each cluster reflects the level of relatedness in each population. Edges between the nodes represent total IBD tract length for pairs of individuals sharing more than $150 \mathrm{cM}$ of total IBD, with edges colored using the log transformed total IBD length. 


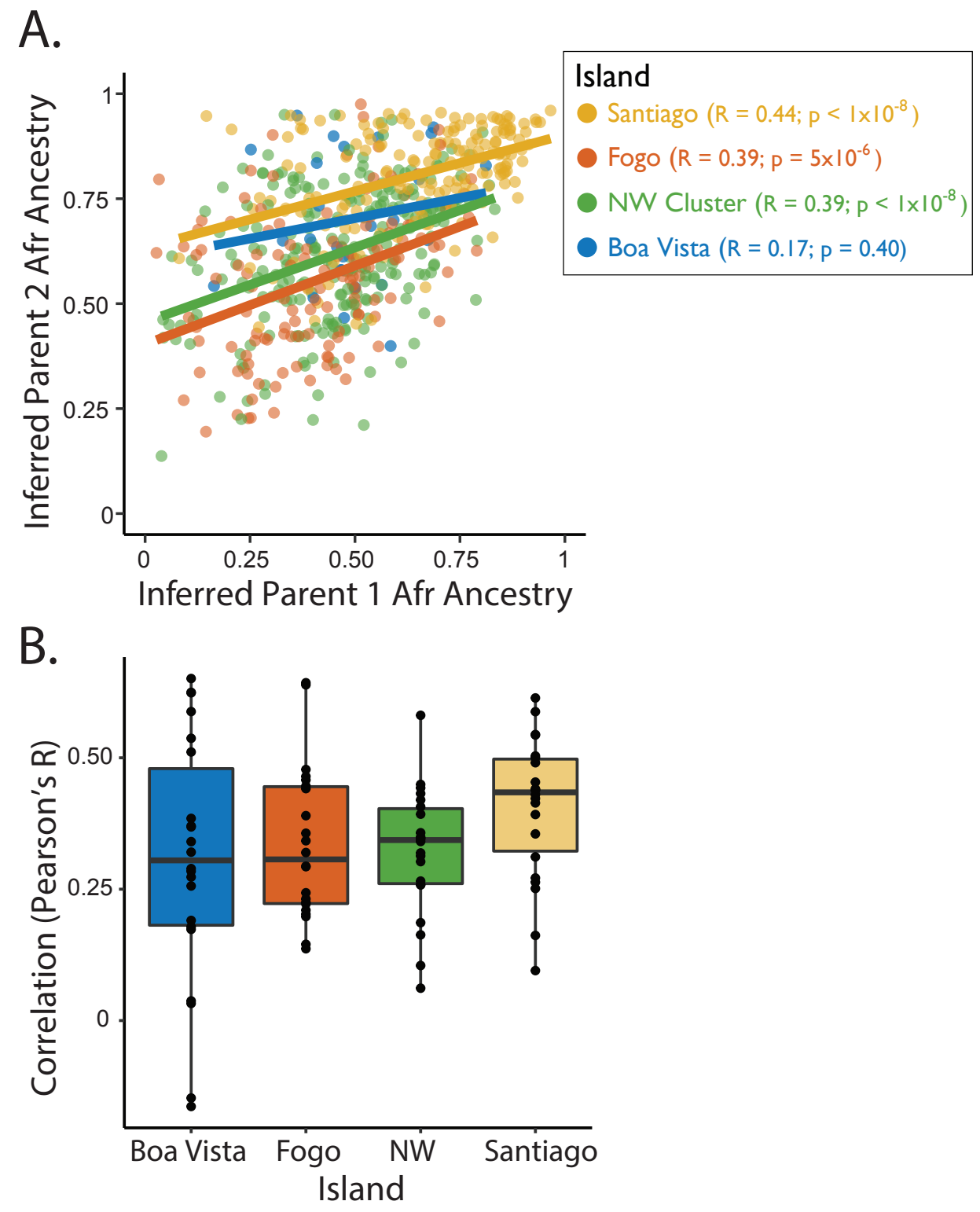

Fig 2 I Correlation between inferred parental ancestries. (A) For each Cabo Verdean individual, the inferred Parent 1 vs Parent 2 ancestry proportions are shown for an example chromosome (Chromosome 7), colored by island. (B) shows the set of correlation coefficients between inferred parental ancestries for each autosomal chromosome. 


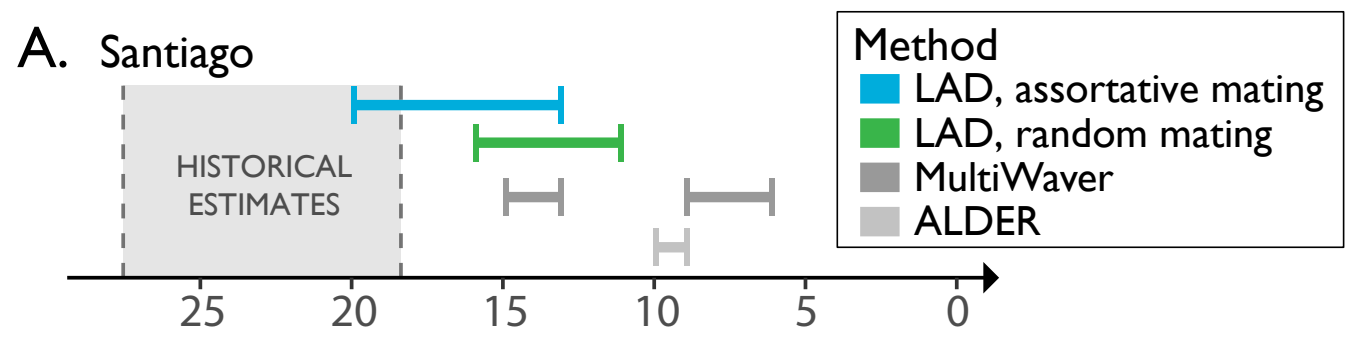

B. Fogo

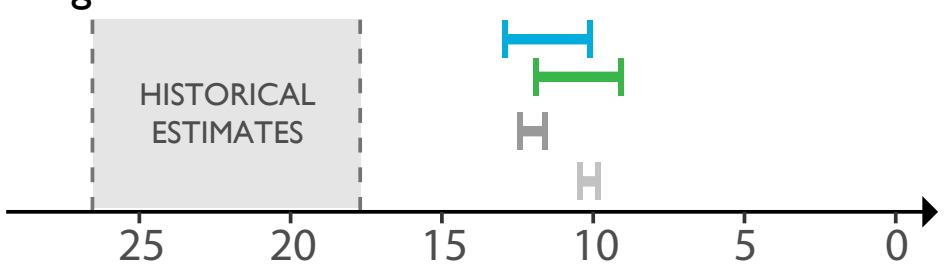

C. NW Cluster

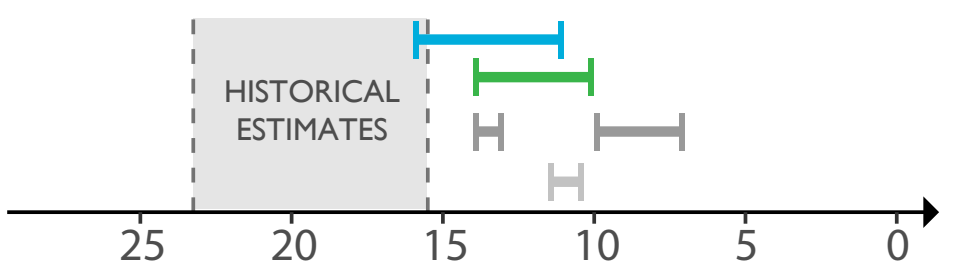

D. Boa Vista

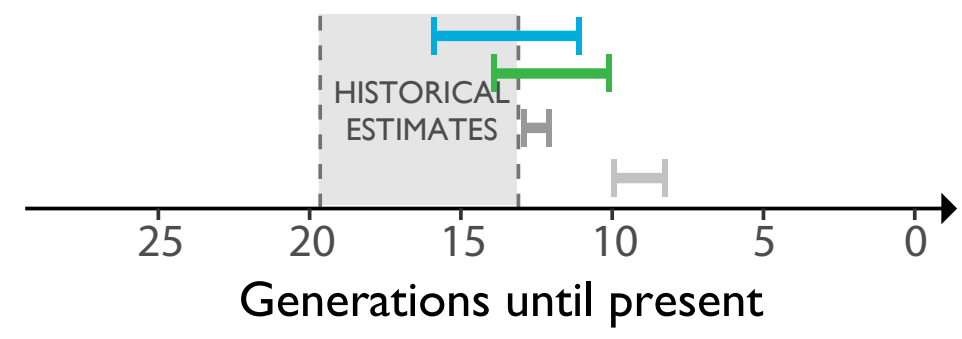

Fig 3 I Timelines of inferred generations of admixture for each island of Cabo Verde. The island regions are listed in order of known settlement timing beginning with Santiago (A), followed by Fogo (B), and then the later migrations to the Northwest Cluster (C) and Boa Vista (D). For each island, admixture timing inferred with population genetic methods are shown in comparison to historical records. Historical estimates of when each island was first settled are shown in generations, based on a range of generation times (20-30 year generation time). Within each LAD-based estimate of admixture timing, the presented interval reflects the range of estimates generated under the assumption of constant migration rate $(\mathrm{m}=0.01$; yielding older estimates) to the assumption of no migration $(\mathrm{m}=0$; yielding more recent estimates). MultiWaver results show the admixture generations inferred under the admixture model selected by MultiWaver, with $0.95 \mathrm{CI}$. 

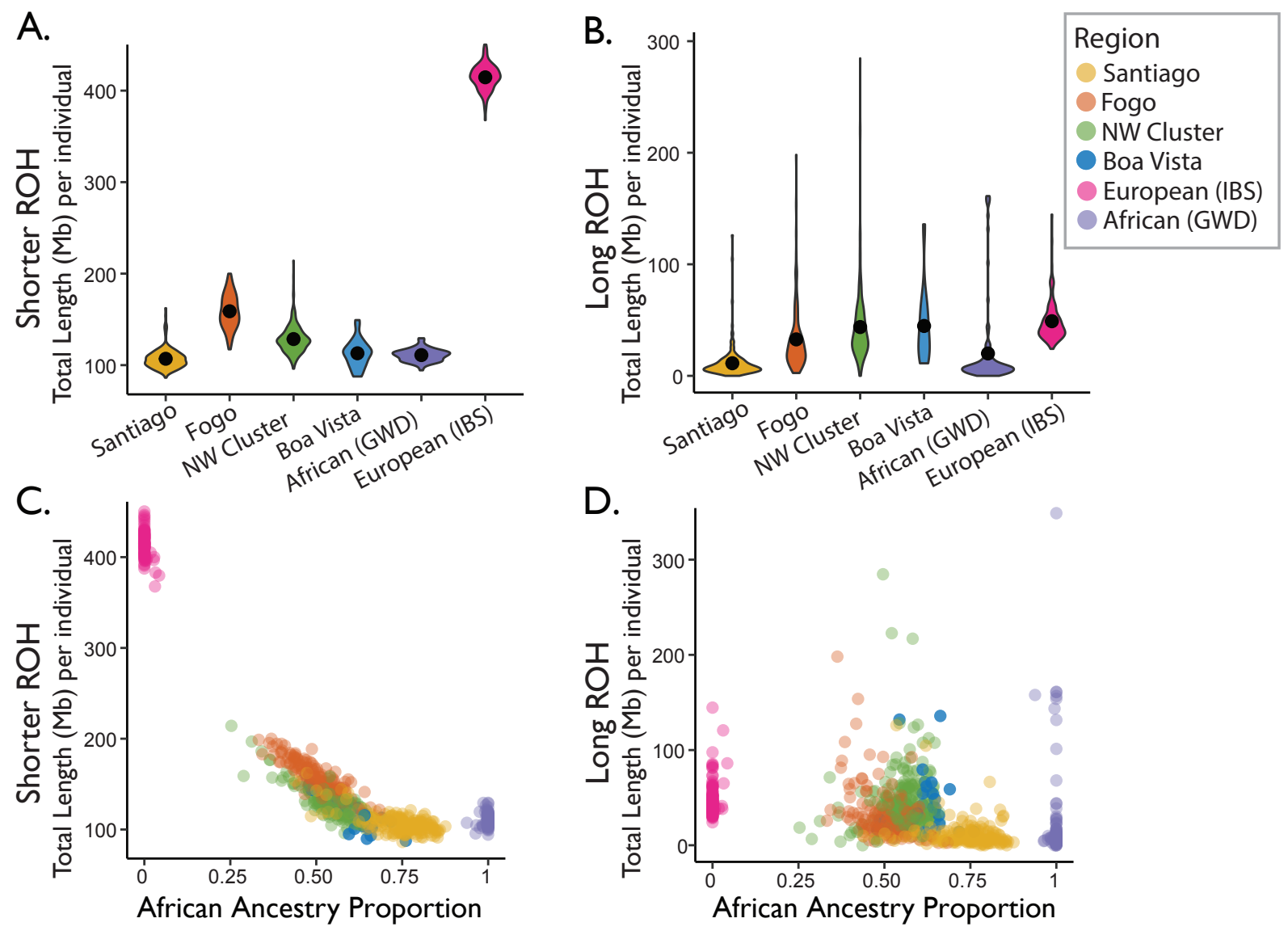

Fig 4 I ROH content by island and in the context of ancestry. (A-B) Violin plots show the population-specific distributions of the total (summed over each genome) length of autosomal ROH per individual. Solid black dots represent the within-population means. (CD) Total length of autosomal $\mathrm{ROH}$ per individual plotted against West African ancestry proportions and colored by population. 

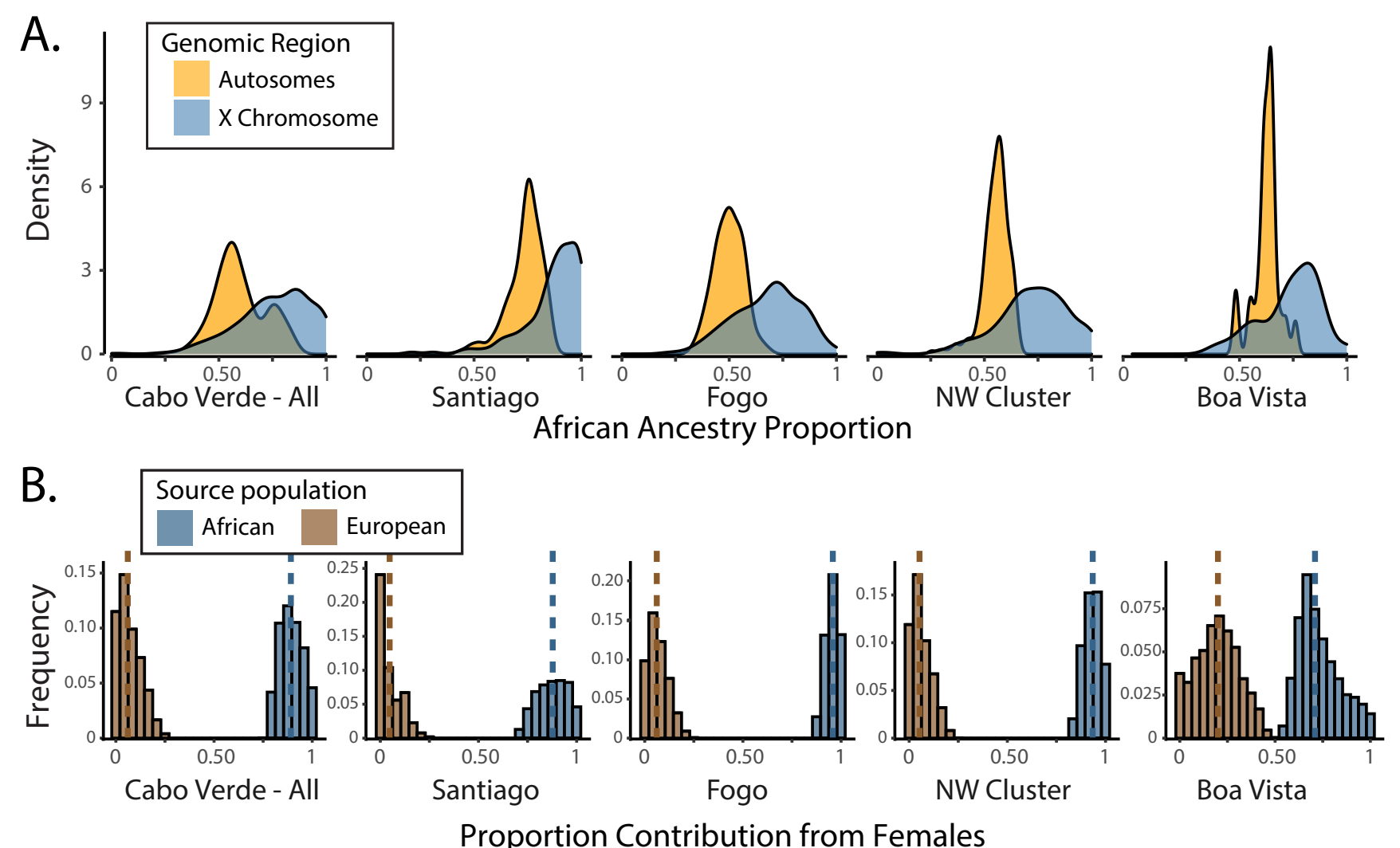

Fig 5 I Sex-biased admixture in Cabo Verde. (A) The distribution of West African ancestry proportion on the autosomes and $\mathrm{X}$ chromosome for each of the island regions, estimated with ADMIXTURE. (B) Under a model of constant admixture over time, the fraction of the total contribution of genetic material originating from females for West African and European source populations. Here, we show the distribution of parameter sets for the smallest $0.1 \%$ of Euclidean distances between the model-predicted and observed $X$ and autosomal ancestry from a grid of possible parameter values. The range of sex-specific contributions from West African and European source populations that produce ancestry estimates closest to those observed in Cabo Verde are shown for Cabo Verde as a whole (left), and then broken down by region, with medians (dashed lines). 


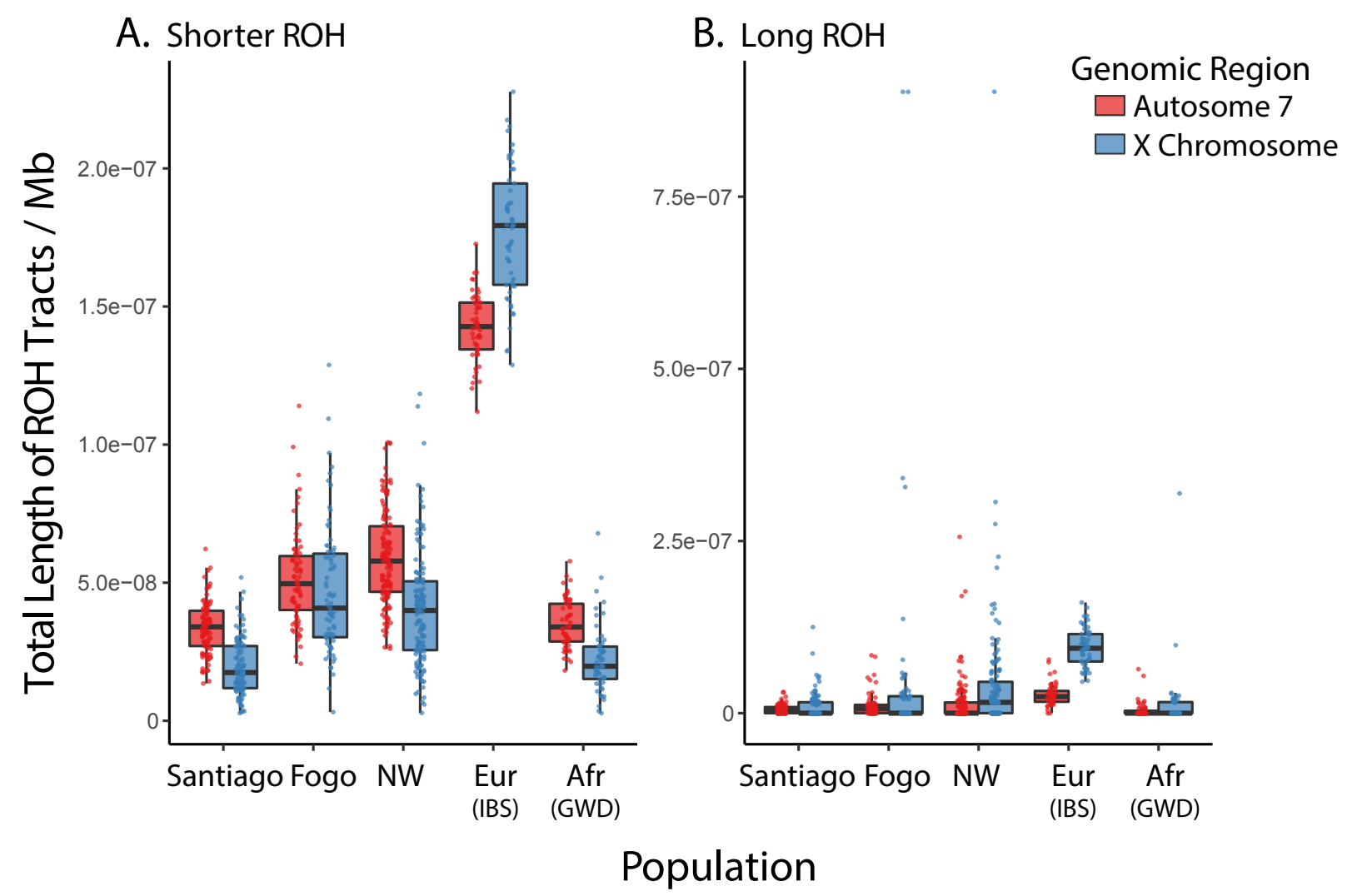

Fig 6 I Autosomal (chromosome 7) vs X chromosome distributions of ROH by class. (A) shows the population-specific distributions of the total length of shorter $\mathrm{ROH}$ per individual on the $\mathrm{X}$ chromosome compared to an autosome. Chromosome 7 was chosen as the autosomal point of comparison, given that it is the autosome most similar in size to the $\mathrm{X}$ chromosome. Totals are shown for females only, so that the same samples are being compared across the $\mathrm{X}$ chromosome and chromosome 7 . Totals are plotted per $\mathrm{Mb}$ to account for slight differences in chromosome lengths. (B) shows the same information for long ROH. 